\title{
CME: Rheumatology (133254): self-assessment questionnaire
}

\author{
Authors: Edited by Elaine Dennison and Tahseen A Chowdhury
}

\section{DOI: $10.7861 /$ clinmed.SAQ.20.6}

SAQs and answers are ONLINE for RCP fellows and collegiate members

\section{Format}

Candidates are asked to choose the best answer from the five possible answers. This best of five format is used in many medical examinations; however, the questions are not intended to be representative of those used in the MRCP(UK) Part 1 or Part 2 Written Examinations.

\section{The answering process}

1 Go to www.rcplondon.ac.uk/SAQ

2 Log on using your usual RCP username and password

3 Select the relevant CME question paper

4 Answer all 10 questions by selecting the best answer from the options provided

5 Once you have answered all the questions, click on Submit

Registering your external CPD credits

Carrying out this activity allows you to claim two external CPD credits. These will be automatically transferred to your CPD diary, where you can review the activity and claim your points.

1. Which of the following statements regarding rheumatoid arthritis is correct?

(a) A positive immunoglobulin $\mathrm{M}$ rheumatoid factor blood test indicates a diagnosis of rheumatoid arthritis.

(b) A quarter of patients with rheumatoid arthritis have positive blood anti-cyclic citrullinated protein antibodies.

(c) Acute phase response markers such as erythrocyte sedimentation rate or $\mathrm{C}$-reactive protein are always elevated in rheumatoid arthritis.

(d) Anti-cyclic citrullinated protein antibodies may be found in the blood many years prior to the onset of symptomatic rheumatoid arthritis.

(e) Rheumatoid arthritis can be excluded as a diagnosis if neither immunoglobulin $\mathrm{M}$ rheumatoid factor nor anticyclic citrullinated protein antibodies can be detected in the blood.

2. A 42-year-old man presented with feeling generally unwell, lethargic, breathless and had lost 1 stone over
4 weeks. Examination showed mild pallor, tachypnoeic, blood pressure of 178/96 mmHg, pulse rate at 94 beats per minute, oxygen saturation at $95 \%$ on room air and a body temperature of $37.8^{\circ} \mathrm{C}$. There were small purpuric rashes at his soles, and mild pedal oedema. Cardiovascular examination showed normal heart sound and no murmur. Lung auscultation revealed scattered crepitations at bilateral lower zones. His haemoglobin was $98 \mathrm{~g} / \mathrm{L}$ (140-180), white blood cell count was $8.1 \times 10^{9} / \mathrm{L}(4-11)$, platelet count was $527 \times 10^{9} / \mathrm{L}(140-450)$, creatinine was $198 \mu \mathrm{mol} / \mathrm{L}(0-135)$, erythrocyte sedimentation rate was $82 \mathrm{~mm} /$ hour (1-7), C-reactive protein was $46 \mathrm{mg} / \mathrm{L}(0-10)$, urine dipstick had blood at $3+$ and trace protein, and chest $X$-ray showed alveolar infiltrates.

\section{Which of the following investigations is the most likely to} confirm diagnosis?

(a) Anti-glomerular basement membrane antibody.

(b) Bronchoscopy and alveolar lavage.

(c) Computed tomography of the thorax.

(d) Myeloperoxidase-antineutrophil cytoplasmic antibodies.

(e) Renal biopsy.

3. Under what circumstance would the cyclophosphamide be preferred over rituximab for inducing remission in patient with severe antineutrophil cytoplasmic antibodyassociated vasculitis?

(a) A completed family.

(b) Previous uroepithelial cancer.

(c) Second relapse of severe antineutrophil cytoplasmic antibody-associated vasculitis with previous exposure to cyclophosphamide.

(d) Severe pancytopenia.

(e) The antineutrophil cytoplasmic antibody-associated vasculitis remained active or progressed despite a course of cyclophosphamide lasting 3-6 months.

4. A 71-year-old man was reviewed in the rheumatology outpatients' department with bilateral shoulder pain, upper back pain and loss of weight for 5 weeks. He had no temporal headache, jaw claudication, blurring of vision nor neurological weakness. Systemic reviews were unremarkable. He had hypertension and hypercholesterolaemia on medications. Physical examination revealed diffuse tenderness at both shoulders and upper thoracic region. Bilateral upper limb proximal muscle power was reduced, associated with pain during examination. Temperature was normal. Erythrocyte 
sedimentation rate on presentation was $50 \mathrm{~mm} /$ hour (1-7), C-reactive protein of $34 \mathrm{mg} / \mathrm{L}(0-10)$. His pain was not responding to prednisolone $15 \mathrm{mg}$ daily.

Which of the following is the most appropriate course of action for this patient?
(a) Arrange positron emission tomography-computed tomography.
(b) Check creatinine kinase level.
(c) Check serum protein electrophoresis.
(d) Increase prednisolone to $40 \mathrm{mg}$ daily.
(e) Initiate treatment with tocilizumab.

5. The pathogenesis of systemic sclerosis relates to which abnormalities?
(a) Autoimmunity.
(b) Autoimmunity, vascular and fibrotic disease.
(c) Paraneoplastic.
(d) Vascular and fibrotic disease.
(e) Vascular disease.

6. What is now the leading cause of death in systemic sclerosis?
(a) Cancer.
(b) Cardiac disease.
(c) Gastrointestinal failure.
(d) Renal failure.
(e) Respiratory complications (interstitial lung disease and pulmonary hypertension).

7. Which of the following is the most appropriate initial approach to bone health in a 60-year-old woman needing daily oral prednisolone for rheumatoid arthritis control?

(a) Daily non-steroidal anti-inflammatory drugs.

(b) Calcium and vitamin D supplementation.

(c) Intravenous zoledronate.

(d) Lifestyle advice.

(e) Rapid assessment of 10-year fracture risk with FRAX ${ }^{\circledR}$ and dual-energy $\mathrm{X}$-ray absorptiometry.

8. Which of the following medications acts primarily on osteoblasts?
(a) Alendronate.
(b) Denosumab.
(c) Etidronate.
(d) Teriparatide.
(e) Zoledronate.

9. What is the most common bone involved with Paget's disease?
(a) Femur.
(b) Pelvis.
(c) Skull.
(d) Spine.
(e) Sternum.

10. Which of the following is a type of tumour that can rarely be associated with Paget's disease?
(a) Chondroma.
(b) Fibroadenoma.
(c) Haemangioma.
(d) Osteoma.
(e) Osteosarcoma

\section{Erratum}

In the previous SAQs, the units for bilirubin in questions 8-10 were presented in $\mathrm{mmol} / \mathrm{L}$, they should have been in $\mu \mathrm{mol} / \mathrm{L}$.

CME hepatology SAQ Answers to the CME SAQ published in Clinical Medicine in September 2020

$\begin{array}{llllllllll}\text { Q1 } & \text { Q2 } & \text { Q3 } & \text { Q4 } & \text { Q5 } & \text { Q6 } & \text { Q7 } & \text { Q8 } & \text { Q9 } & \text { Q10 } \\ \text { (d) } & \text { (b) } & \text { (c) } & \text { (d) } & \text { (c) } & \text { (e) } & \text { (c) } & \text { (c) } & \text { (c) } & \text { (d) }\end{array}$

